\title{
"The damage I could do..."- Qualitative evaluation of a low-fidelity medication administration simulation that generates error as a learning experience for pre-registration nursing students
}

\author{
Sinead Helyar ${ }^{1}$, Peter Griffiths ${ }^{1}$, I an J Norman ${ }^{2}$ \\ 1. Faculty of Health Sciences, University of Southampton, Southampton, UK. 2. Florence Nightingale School of Nursing, \\ Kings College, London, UK.
}

Correspondence: Sinead Helyar. Address: Emergency Department, Kings College Hospital, Denmark Hill, London, UK. E-mail: sinead.helyar@nhs.net

Received: February 27, 2013

Accepted: May 6, 2013

Online Published: September 10, 2013

DOI : $10.5430 /$ jnep.v4n2p11

URL: http://dx.doi.org/10.5430/jnep.v4n2p11

\begin{abstract}
Background: In recent years, nurse education has emphasised the development of high cost, high-fidelity simulations because they are considered to provide a more realistic learning experience. Integral is the facility to commit and learn from error. Error may provide a mechanism to make low cost, low-fidelity simulations more psychologically realistic and salient. We developed a web based medication administration simulation which included all essential cognitive elements of medication administration task, but was "low-fidelity" in engineering terms of its portrayal of the physical world. The simulation incorporated conditions designed to generate high rates of medication administration error. The purpose of this study was to determine if the low cost low-fidelity simulation provided a salient, effective and psychologically realistic learning experience for pre-registration nursing students over the long term.
\end{abstract}

Methods: Individual qualitative interviews were conducted with 12 pre-registration nursing students, from one UK university, from a group of 49 students who had received the simulation as trial participants 2-years previously. The interviews were analysed using thematic analysis.

Results: Most participants were able to reflect on their experiences of the simulation in depth, and considered the simulation to be a valuable component to their learning. The simulation encouraged students to question their practice and highlighted their vulnerability to medication administration error. It reinforced both the importance of completing checking procedures and the potential consequences of making an error in practice. Most students believed the lowfidelity simulation provided a psychologically realistic representation of medication administration, which was underpinned by the negative emotional reactions to making an error, for example, guilt.

Conclusions: Low-fidelity web based simulations can provide a sufficiently realistic psychological representation of medication administration to produce a salient, effective learning experience over the long-term. Low-fidelity simulations can provide a low cost alternative to high-fidelity simulations. Making error in a simulation should be transformed from a useful but passive by-product into an active component of the simulation learning approach.

\section{Key words}

Low-fidelity simulation, Patient safety, Drug administration error, Qualitative research

Published by Sciedu Press 


\section{Introduction}

Simulation is an integral teaching approach for nurse education to develop and support intellectual and clinical skills ${ }^{[1]}$, reflective practice ${ }^{[2]}$ and assess competency ${ }^{[3,4]}$. Simulation is an artificial representation of an aspect of clinical practice, which can encompass an environment, scenario or clinical task for students to interact with. Simulation is an activity which "mimics" reality to enable students to learn ${ }^{[5,6]}$. This mimicry combines and supports links between theory and practice ${ }^{[7-9]}$. Simulation comprises various methods and levels of fidelity to enable a student to 'suspend belief' and substitute the simulation for clinical practice to learn predefined skills ${ }^{[10]}$.

Fidelity is the extent to which a simulation accurately reflects a real life situation and is commonly categorised as "high", "medium" or "low". Higher fidelity simulations incorporate more realistic features ${ }^{[11]}$. Fidelity can be divided into engineering, psychological ${ }^{[12]}$ and environmental fidelity ${ }^{[13]}$. Engineering and environmental fidelity refer to the degree to which the simulation depicts the real environment, equipment or physical characteristics of the represented task. Psychological fidelity denotes the extent to which task skills are captured in the simulation, or more simply, how psychologically realistic the student finds the simulation. In line with technological advances, interest has focused upon the development of more complex, high-fidelity methods ${ }^{[2,12,14]}$. However, a significant concern with high-fidelity simulation is its high cost, particularly given the current financially austere times in many countries.

Considerable investment is required to integrate high-fidelity simulation ${ }^{[15,16]}$ into the nursing curriculum, and it has been classed as a costly ${ }^{[17,18]}$ and complex resource ${ }^{[8]}$. For example, high-fidelity simulation mannequins can be 30 times more expensive as compared to low-fidelity simulation mannequins ${ }^{[14]}$. Costs result not just from the purchase and development of the simulation, but also encompass time, space, training and administration ${ }^{[19]}$. The implementation and integration of a high-fidelity simulation laboratory at one institution cost over $\$ 300,000$, with additional maintenance costs ${ }^{[2]}$. The cost can potentially render high-fidelity simulations prohibitive.

The advantage of high-fidelity simulations is the level of realism achieved ${ }^{[2,12]}$ because it the aids preparation for clinical practice $^{[11]}$. However, high engineering and environmental fidelity is insufficient if not paired with appropriate psychological fidelity ${ }^{[13]}$. Psychological fidelity or psychological realism is the critical component which goes to the core purpose of a simulation and its educational outcomes. This can be maximised by the inclusion of scenarios that replicate the demands of clinical practice ${ }^{[13]}$. One cost-effective strategy to strengthen the effectiveness and salience of low-fidelity simulation learning is to maximise psychological fidelity or realism for students. One potential mechanism to achieve this is through the integration and experience of error.

The importance of experiencing and learning from error is extensively documented within simulation literature ${ }^{[11,20]}$. Within simulations, making and experiencing error is considered to be a "powerful educational experience" which can enhance professional competency and foster a "correct attitude", to minimise real-life errors ${ }^{\text {[21] }}$. Errors may increase the salience of theory by making clear that precautionary actions, such as those taught as theory, are fundamental for safe professional clinical practice. Within most simulations, the experience of error is employed as a useful by-product of the simulation learning experience. However, some suggest error should be actively incorporated in the simulation learning scenario. Simulation learning enables students to “deliberately” experience and learn through failure, a strategy unethical in clinical practice ${ }^{[20]}$. Such an approach affords students the opportunity to learn new information and problem-solve real-life situations in a safe and structured setting ${ }^{[21]}$. Error can generate an emotional response that can be used to highlight the possibility of error and the need for the application of error preventing strategies. Error and mistakes are therefore proactively used as a learning tool to improve or prepare for clinical practice. The experience of error is potentially pivotal in forging links between theory and clinical practice.

We developed a web based medication administration simulation which comprised all essential cognitive elements of medication administration task, but was "low-fidelity" in engineering terms of its portrayal of the physical world. The simulation incorporated conditions designed to generate high rates of medication administration errors. We hypothesised 
that the active incorporation of error into a low-fidelity simulation can instil higher levels of psychological fidelity or realism and make the simulation into a more salient and effective learning tool over the long term. This paper reports a qualitative exploration of the long-term impact of participating in this novel educational simulation.

\section{Methods}

\subsection{Simulation overview}

The simulation was a low-fidelity web based oral medication administration simulation. Medication administration was selected because of the extent of medication administration error in clinical practice ${ }^{[22,23]}$. The simulation was manipulated to increase the chance of a "right drug, wrong error", a medication administration error common in practice ${ }^{\text {[24] }}$ and in some studies, the most prevalent ${ }^{[25]}$. This was achieved by exploiting the robust psychological phenomenon of "change blindness" [26] "Change blindness" results from selective attention/inattention and is the phenomenon whereby an observer fails to notice changes to non-attended items of a visual scene when they are distracted, however transiently.

Within the simulation, participants were presented with the details and photographs of three pseudo patients, situated in their own individual bed space. Each patient had a link to a name band, which comprised all the patient's identification and background information to enable accurate checking, and a link to their medication chart. Participants were informed that all medications were suitable to administer as prescribed, and were asked to administer medications to one "target" patient.

When the "target" patient's medication chart was opened, participants were able to select and dispense the prescribed medications and place them into a cup. The medication chart opened in a new tab across the whole screen to obscure the participant's view of the patients. Behind the medication chart, the "target” patient changed bed space with a physically similar "decoy" patient. The patients retained their own name band to enable accurate checking. The physical similarity of the patients was determined in a preceding titration phase.

When the participant finished dispensing medications into the cup, they closed the medication chart and returned to view the patients and administer the medications to the "target" patient. The "right drug, wrong patient" error occurred when the participant administered medications to the "decoy" patient, now in the "target” patient’s original bed space. Participants were required to administer medications to ten "target" patients within 15 minutes. At the end of the simulation, participants were informed of their medication administration accuracy rates and the accuracy rates of others immediately postcompletion.

\subsection{Sample and setting}

This study was conducted at a London University between April 2008 and April 2010. A convenience sample of 49 first year pre-registration nursing students completed the simulation, as part of a randomised control trial investigating different medication administration teaching methods. All students who completed the simulation were approached via email and as a group in class with a request to participate in this follow-up study 18-24 months later, whilst in their third and final year of pre-registration study. The students were asked to participate in qualitative interviews regarding the long-term outcomes of their medication administration learning experience. In total, 12 students responded and agreed to be interviewed. One student was male and 11 were female. This study reports the interview phase of the study.

\subsection{Design and procedure}

The interviews comprised semi-structured qualitative interviews, based on a loose and flexible interview structure. The interviews were completed by one interviewer and typically lasted between 30 - 50 minutes. The interviews commenced with general questions about the participant's medication administration education and narrowed to questions about the use and effectiveness of the medication simulation as an education method. Example questions included, "Can you go through how you were taught medication administration in university and in clinical practice?”, "How easily do you 
remember completing the medication simulation?”, “Describe how you went through the simulation?”, “Did you make any errors in the simulation?”

\subsection{I nterview analysis}

The interviews were analysed using thematic analysis ${ }^{[27]}$. The interviews were transcribed and analysed for themes by one researcher and the themes were reviewed by two further researchers. Theme identification was driven by the theoretical foundations of the research and from what emerged from within the interview data itself. Themes were generated using the literal semantic meanings of what was articulated in the interview data and through latent interpretations. Latent interprettation refers to the underlying assumptions and meanings that underpinned what was articulated in the interviews.

The researcher commenced by familiarising themselves with the interview data and produced a list of 'codes', or potential areas of interest. The codes were iteratively examined to identify patterns between the codes that could generate broader themes. The generated themes were also examined to identify links and contradictions. These were iteratively revised and linked back to the initial codes to identify themes to be removed, merged or subdivided, and if the themes identified reflected the interview data. This process was iteratively conducted and concluded when no further themes, links and contradictions could be identified.

\subsection{Ethical considerations}

Approval was gained through the university ethics board before completion of the simulation and at the commencement of the interview phase of the study. Participants completed the simulation as part of their nursing curriculum and therefore informed consent was only obtained for the acquisition of simulation results and subsequent data collection activities. Informed consent was separately obtained from participants immediately prior to interview participation.

\section{Results}

All of participants remembered completing the simulation two years earlier. Two participants stated they remembered the simulation vaguely and were not able to discuss the simulation in depth. The remaining participants were able to discuss and reflect upon their experience in depth. Eleven of the twelve participants recalled making an error. The themes identified in the interview analysis highlighted that most participants considered the simulation to be valuable to their learning. The themes predominantly related to the experience of error and how this impacted upon their learning of safe medication administration practice, the emotional impact of making an error, and how realistic the participants perceived the simulation to be.

\section{Themes}

\section{“Shocked when I didn’t get them all right!” - Surprise and questioning current practice}

Ten of the eleven participants who made an error were surprised to have done so. The active experience of error enabled participants to recognise they could be the nurse to make an error, something they had not previously considered. Error was no longer just a theoretical possibility, but intrinsic to them and possible in their clinical practice.

"I thought I definitely got them all right and was a bit shocked when I realised that I didn't get them all right..." (participant 3)

“It reinforced it in my mind and I thought okay look, you’ve had your chance, don’t do it again...”. (participant7)

"I remember being a bit peeved that I made an error, and I then when I looked back on it, it was like the whole point... it really made you aware of how important it is not to make errors” (participant 8) 


\section{"Still with me today" - Bridging the theory-practice gap}

Nine participants discussed how the simulation and specifically making an error reinforced the importance of theory and medication checking procedures, in particular, in terms of their intended future use of checking whilst in clinical practice. For example,

“They (errors) were ones where I obviously hadn’t realised how important some of the theory is, obviously about identification and things...We had always been told, but obviously the simulation brought it to my attention just that much more.” (participant 7)

For some participants, the experience of error not only reinforced, but helped form and shape their understanding of theory in terms of checking procedures within practice. For example,

"I think that is when I really started really checking and double checking”. (participant 2)

"Knowing I need to check that the patient is right and the tablet is right arose from those mistakes made." (participant 5)

Participants expanded their learning beyond the specific error made to medication administration error in general. Making an error acted as a trigger to alert participants to the importance of safe medication administration and helped to consolidate a culture of safe medication administration practice. For example;

“It made you think more about things that could go wrong” (participant 1)

"It developed my knowledge and from that I learnt. For me...it just made me much more aware how to be safe ...it really confirmed how important safety is. You have to do everything you possibly can to ensure your practice is safe. ... it is something that I took away from that and still practice, is still with me today”. (participant 4)

"Knowing how easy it is to make a mistake, I think, it means then you don’t then become complacent" (participant 8)

“Makes you think that you shouldn’t just rely on things staying the same”, (participant 9)

The highlighted importance of checking procedures stems from the knowledge that they will make an error if they are not careful. The simulation and error demonstrated the correct medication administration is a complex, multi-dimensional task, not previously considered by participants.

\section{“The damage I could do...” - Appreciating risk in clinical practice}

Ten participants recognised and feared that if they make an error in a simulation, they can make an error in practice, and were concerned about the potential harm to patients from their errors in practice. Although errors made in the simulation were specific and would not result in an adverse consequence for the patient, many participants extrapolated the error to encompass other types of error and in terms of the worst case scenario of killing the patient.

“That was a shock - the damage I could do" (participant 4)

“You could have potentially killed a patient!” (participant 6)

“It made me feel, oh my god, how many patients might I have killed?” (participant 11) 
The simple error exploited the participants' fundamental fears and concerns as a nurse of causing harm to their patient. This demonstrates that it is not the actual consequence, but the fear of potential harm can be exploited for learning in a simulation setting. To participants this underpinned the importance of theory and checking procedures as a protective measure against error and made the simulation a salient and effective learning experience.

For some participants, the experience of error increased their awareness of their professional responsibilities to their profession and their patients. It highlighted an error can have personal consequences for them as individual practitioners and underlines the gravity of the nursing role:

"I think it highlights that I am accountable” (participant 1)

"As a nurse, bearing in mind the professional code of practice you operate under, you sign up for... you have to make sure your practice is safe... it really conserved it, embedded it”. (participant 4)

"But yeah when you sort of relate the seriousness of the test to real life, you obviously would get into a lot of trouble...” (participant 12)

Participants understood their future responsibilities which both scared them and motivated them to be accurate nurses and to complete checking procedures.

\section{“I felt horrible”-Emotional reaction}

Eleven participants experienced a negative emotional reaction to making error, including: "guilt”, "shock”, "feeling sick”, "scared”, "stupid”, “disappointed” and "horrible”.

Participants were shocked that they made an error and experienced difficulty reconciling their pre-simulation assumption that they would be safe medication administrators to making an error. The emotional reaction provided an added dimension to their learning, made the simulation easier to recall and more salient. Interestingly, one of the minority of participants who did not support the simulation also experienced a negative emotional reaction to error, which underlines the potential impact of the experience of error.

“Erm gutted, you just kinda go ahhhh oh no!! It felt awful.” (participant 10)

\section{“Like the real thing...”- Psychologically realistic}

Nine participants stated they considered the low-fidelity design of the simulation to be a psychologically realistic representation of clinical practice. They were positive about the simulation and considered the simulation effectively incorporated key cognitive elements the medication administration task, in relation to lay out, ability to complete checks and use and design of medication chart. For example,

"I think it did it really well. I wouldn't know how to improve it as a computer simulation. All the points of the theory, ie, the right drug, right dose etc., were covered. There were multiple options and scenarios changed as they kind of would on the ward.” (participant 5)

"You could see it was just an ordinary drug round". (participant 6)

Two participants felt the simulation lacked the interactivity necessary to replicate the communication between nurses and patients. They believed this was necessary to establish if the medication should be administered. Two participants discussed the need to freeze reality during the simulation, however, this is inherent to all simulations as participants know they are not real ${ }^{[28]}$, and did not negate the learning experience. 
"In my head I was thinking it was not quite realistic...it kind of reminded me that no, I do need to take it seriously no matter what happens” (participant 7)

\section{Discussion}

The aim of this study was to determine whether a low cost, low-fidelity web based simulation could provide a salient, effective and psychologically realistic learning experience for pre-registration nursing students. The results of the qualitative interviews demonstrate that the simulation was salient over the long term. Most participants were able to discuss and reflect upon their experience and learning in depth two years later. The experience of error in the simulation was pivotal to the participant's learning. It helped participants to question their current practice and highlighted their vulnerability to make errors if theory and checking procedures are not adhered to. Learning was expanded beyond the narrow confines of the actual error made to error in general. This culminated for some in the realisation that through error, they could "kill" a patient. This forged and reinforced the importance of the theory underpinning safe medication administration practice and checking procedures. To this end, the simulation successfully served as a "hybrid" of theory and practice.

The simulation also provided a psychologically realistic representation of medication administration and error. This was demonstrated by the ease in which participants could interact with the simulation as an "ordinary drug round". In addition, the participants' emotional responses to the medication administration errors made in the simulation were similar to those expressed by qualified nurses who made medication errors in clinical practice. Reported feelings on making a medication administration error include guilt, upset and terror ${ }^{[29]}$, which were mirrored by participants in this study. This is supported by other studies ${ }^{[30,31]}$. The participants were student nurses and as such it is unlikely that they would have previously made medication errors which may have contaminated their reactions in the simulation. The similarity of reaction exemplifies the connection between making error in the simulation and in clinical practice, and demonstrates the low cost low-fidelity simulation generated a psychologically realistic experience of clinical practice.

This study adds to the current research base which does not unanimously support the use of more complex and expensive simulations ${ }^{[14,17]}$. In a cost-utility analysis of medium to high fidelity simulation mannequins, it was identified that medium-fidelity mannequins achieved commensurate scores to high-fidelity mannequins on clinical reasoning, knowledge acquisition and student satisfaction, at one fifth of the cost ${ }^{[32]}$. The similarity of outcomes despite differences in cost emphasises the importance of calculating simulation costs to educational outcomes, because costs do not always correlate with educational outcomes. Criteria for simulation selection should be based on fitness for purpose; to maximise education outcomes with the minimum cost ${ }^{[8]}$. Simulations should be produced that are "good enough" and lower-fidelity simulations are capable of providing an effective learning experience ${ }^{[33]}$. However, given the importance of realism for effective simulation learning, low-fidelity simulations need to be sufficiently realistic and authentic for purpose. This study highlights that the effective introduction of psychological realism, through the use of error, can make a low cost, low fidelity simulation sufficiently realistic.

\section{Methodological considerations}

The study was limited to one site and the sample was self selecting, however the commonality of responses between participants suggests these results could be extrapolated to a larger number of students. A further limitation is we did not compare the simulation to one in which we did not deliberately manipulate error. However, the students' responses suggest the experience of error was central to their learning experience. Participants were interviewed two years later and may have forgotten salient aspects of the experience which nonetheless were incorporated into their learning. In addition, experiences in between the simulation and the interviews may make it difficult for participants to identify the specific role of the simulation in their medication administration education. Further research includes testing the novel simulation on larger numbers of participants and its effect on actual medication administration practice. 


\section{Conclusion}

This study highlights how the incorporation and experience of error within a low cost, low-fidelity web based simulation can provide a salient, effective and psychologically realistic learning experience for pre-registration nursing students over the long term. The participants were interviewed two years after completing the simulation. The deferred evaluation is a model that should be encouraged in the evaluation of simulation education. It enables the comprehensive assessment of the actual transfer of simulation learning into clinical practice and the retention of learning over the long-term; the goal of simulation learning. It also identifies if the simulation experience is not retained and learning outcomes over the long term are not achieved. This study demonstrates that low-fidelity simulations can be psychologically realistic and supports the assertion that simulations only need to be "good enough" for effective learning ${ }^{[33]}$. The use of error enabled the lowfidelity simulation to achieve a greater degree of psychological realism and salience. This approach can be an effective and low-cost alternative to high-fidelity simulations. In the current financially austere times, more emphasis should be placed on the cost-utility of simulation learning. This study demonstrates that learning through the active application of error is one approach to maximise educational outcomes, whilst simultaneously minimising costs. The active utilisation of error should be transformed from a passive, but welcome by-product of simulation learning into an active component of the simulation learning approach.

\section{Acknowledgements}

Brent Cunningham. Information Technology Officer, Florence Nightingale School of Nursing. Kings College London. London. UK

\section{References}

[1] Long, R. E. Using Simulation to Teach Resuscitation: An Important Patient Safety Tool. Critical Care Nursing; Clinics of North America. 2005; 17(1): 1-8. PMid:15749395 http://dx.doi.org/10.1016/j.ccell.2004.09.001

[2] Garrett, B. M., MacPhee, M. and Jackson, C. Implementing high-fidelity simulation in Canada: Reflections on 3 years of practice. Nurse Education Today. 2011; 31(7): 671-676. PMid:21075494 http://dx.doi.org/10.1016/j.nedt.2010.10.028

[3] Feingold, C.E., Calaluce, M., Kallen, M.A. Computerized patient model and simulated clinical experiences: evaluation with baccalaureate nursing students. The Journal of Nursing Education. 2004; 43(4): 156-163. PMid:15098909

[4] Harder, B.N. Use of simulation in teaching and learning in health sciences: a systematic review. The Journal of Nursing Education. 2010; 49(1): 23-28. PMid:19731886 http://dx.doi.org/10.3928/01484834-20090828-08

[5] Waxman, K.T. The Development of Evidence-Based Clinical Simulation Scenarios: Guidelines for Nurse Educators. Clinical Simulation Scenarios. Journal of Nursing Education. 2010; 49(1): 29-35. PMid:19810672 http://dx.doi.org/10.3928/01484834-20090916-07

[6] Kneebone, R. Simulation in surgical training: educational issues and practical implications. Medical Education. 2003; 37(3): 267-277. http://dx.doi.org/10.1046/j.1365-2923.2003.01440.x

[7] NMC. Supporting direct care through simulated practice learning in the pre-registration nursing programme. 2007. Retrieved from http://www.nmcuk.org/Documents/Circulars/2007circulars/NMCcircular36_2007.pdf (Accessed March 2012)

[8] Kneebone, R.L., Nestel, D., Vincent. C., Darzi. A. Complexity, risk and simulation in learning procedural skills. Medical Education. 2007; 41(8): 808-814. PMid:17661889 http://dx.doi.org/10.1111/j.1365-2923.2007.02799.x

[9] McCaughey, C.S., Traynor, M.K. The Role of Simulation in Nurse Education Training. Nurse Education Today. 2010; 30(8) 827-832. PMid:20483188 http://dx.doi.org/10.1016/j.nedt.2010.03.005

[10] Reilly, A., Spratt, C. The perceptions of undergraduate student nurses of high fidelity simulation-base learning: a case report from the University of Tasmania. Nurse Education Today. 2007; 27(6): 542-550. PMid:17069935 http://dx.doi.org/10.1016/j.nedt.2006.08.015

[11] Butler, K.W., Veltre, D.E., Brady, D. Implementation of Active Learning Pedagogy Comparing Low-Fidelity Simulation Versus High-Fidelity Simulation in Paediatric Nursing Education. Clinical Simulation in Nursing. 2009; 5(4): e129-e136. http://dx.doi.org/10.1016/j.ecns.2009.03.118

[12] Berragan, E. Simulation: An effective pedagogical approach for nursing? Nurse Education Today. 2011; 31(7): 660-663. PMid:21334797 http://dx.doi.org/10.1016/j.nedt.2011.01.019 
[13] Beaubien, J.M., Baker, D.P. The use of simulation for training teamwork skills in health care: How low can you go? Quality \& Safety in Health Care. 2004; 13: 51-56. http://dx.doi.org/10.1136/qshc.2004.009845

[14] Kardong-Edgren, S., Anderson, M., Michaels, J. Does Simulation Fidelity Improve Student Test Scores? Clinical Simulation in Nursing Education 2007; 3 e21-e24 (accessed April 2012) http://dx.doi.org/10.1016/j.ecns.2009.05.035

[15] Gaba, D, M. The future vision of simulation in health care. Quality Safety in Health Care. 2004; 13: 2-10. http://dx.doi.org/10.1136/qshc.2004.009878

[16] Zigmont, J.J., Kappus, L.J., Sudikoff. S.N. Theoretical Foundations of Learning Through Simulation. Seminars in Perinatology. 2011; 35 (2): 47-51. PMid:21440810 http://dx.doi.org/10.1053/j.semperi.2011.01.002

[17] Alinier, G., Hunt, W.B., Gordon, R. Determining the value of simulation in nurse education: study design and initial results. Nurse Education in Practice. 2004; 4(3): 200-207. http://dx.doi.org/10.1016/S1471-5953(03)00066-0

[18] Lane J.L., Slavin S., Ziv, A. Simulation in medical education: a review. Simulation \& Gaming. 2001; 32(3): $297-314$. http://dx.doi.org/10.1177/104687810103200302

[19] Issenberg, S.B. The scope of simulation-based healthcare education. Simulation in Healthcare: The Journal of the Society for Simulation in Healthcare. 2006; 1(4): 203-208. PMid:19088590 http://dx.doi.org/10.1097/01.SIH.0000246607.36504.5a

[20] Kneebone, R.L., Scott, W. Darzi, A. Horrocks, M. Simulation and clinical practice: strengthening the relationship. Medical Education. 2004; 38: 1095-1102. PMid:15461655 http://dx.doi.org/10.1111/j.1365-2929.2004.01959.x

[21] Ziv, A., Ben-David, S., Ziv, M. Simulation based medical education: An opportunity to learn from errors. Medical Teacher. 2005; 27(3): 193-199. PMid:16011941 http://dx.doi.org/10.1080/01421590500126718

[22] Department of Health -Building a Safer NHS, Improving Medication Safety 2004. Retrieved from http://www.dh.gov.uk/en/Publicationsandstatistics/Publications/PublicationsPolicyAndGuidance/DH_4071443. Accessed May 2009

[23] Picone, D., Titler, M., Dochterman, J., Shever, L., Kim, T., Abramowitz, P., Kanak, M., Qin, R. Predictors of Medication Errors Among Elderly Hospital Patients. American Journal of Medical Quality. 2008; 23(2): 115-127. PMid:18305099 http://dx.doi.org/10.1177/1062860607313143

[24] Hicks, R., Becker, S., Krenzischeck, D., Beyea, S. Medication errors in the PACU: a secondary analysis of MEDMARX findings. Journal of PeriAnesthesia Nursing. 2004; 19(1): 18-28. PMid:14770379 http://dx.doi.org/10.1016/j.jopan.2003.11.007

[25] Ito, H., Yamazumi. S. Common types of medication errors on long-term psychiatric care units. International Journal Quality Health Care. 2003; 15(3): 207-212. http://dx.doi.org/10.1093/intqhc/mzg038

[26] Levin, D.T., Simons, D. J. Failure to Detect Changes to Attended Objects in Motion Pictures. Psychonomic Bulletin and Review. 1997; 4(4): 501-506. http://dx.doi.org/10.3758/BF03214339

[27] Braun, V., Clarke, V. Using thematic analysis in psychology. Qualitative Research in Psychology. 2006; 3(2): 77-101. http://dx.doi.org/10.1191/1478088706qp063oa

[28] Lasater, K. High-Fidelity Simulation and the Development of Clinical Judgment: Students’ Experiences. Journal of Nursing Education. 2007; 46(6): 269-76. PMid:17580739

[29] Mayo, A. and Duncan, D. Nurse perceptions of medication errors: what we need to know for patient safety. Journal of Nursing Care Quality. 2004; 19(3): 209-217. PMid:15326990 http://dx.doi.org/10.1097/00001786-200407000-00007

[30] Gladstone, J. Drug administration errors: a study into the factors underlying the occurrence and reporting of drug errors in a district general hospital. Journal of Advanced Nursing. 1995; 22: 628-37. PMid:8708180 http://dx.doi.org/10.1046/j.1365-2648.1995.22040628.x

[31] Santos, J.O., Silva, A.E.B.C., Munari, D.B., Miasso, A.I. Feelings of nursing professionals after the occurrence of medication errors. Acta Paulista de Enfermagem. 2007; 20(4): 483-8.

[32] Lapkin, S., Levett-Jones, T. A cost-utility analysis of medium vs. high-fidelity human patient simulation manikins in nursing education. Journal of Clinical Nursing. 2011; 20(23-24): 3543-52. PMid:21917033 http://dx.doi.org/10.1111/j.1365-2702.2011.03843.x

[33] Kneebone, R.L. Simulation. In Fry, H. Kneebone, R, (Eds). Surgical Education: Theorising an Emerging Domain. Advances in Medical Education. Springerlink. 2011; 37-54. 